\title{
Proteína S libre en embarazadas normales: comparación entre dos métodos
}

\author{
Patricia Fardella B, Mauro Parra C, G uillermo Conte L, \\ Claudio Flores $P$, Hernán Muñoz S, Lilian Soto $S$, \\ Marianela Cuneo Va, Carmen Mallea Pa, María Beatriz \\ Retamales $\mathbf{M}^{\mathrm{b}}$, Sofía Peña $\mathbf{R}^{\mathrm{b}}$, Constanza 0 jeda $\mathbf{H}^{\mathrm{b}}$. \\ Free protein $S$ (PS) in normal \\ pregnancy: A comparison between \\ two analytical methods
}

Background: Pregnancy is a physiological hypercoagulable state with an increased incidence of thromboembolic phenomena. There is an increase in the concentrations of most clotting factors, a decrease in concentration of some of the natural anticoagulants and reduced fibrinolytic activity. Changes in PS levels have also been reported. Aim: To establish referral range values of functional PS and free PS antigen, during the second $\left(2^{\text {nd }} \mathrm{T}\right)$ and third trimester $\left(3^{\text {rd }} \mathrm{T}\right)$ of normal gestation. Patients and methods: Forty one normal pregnant women were included in our study, 20 during the $2^{\text {nd }} \mathrm{T}$ (22-24 weeks) and 21 during the $3^{\text {rd }} \mathrm{T}$ (29-38 weeks). Functional PS was measured by a clot based test and free PS antigen by ELISA. Results: Free PS Antigen was $65.8 \pm 18.3 \%$ during the $2^{\text {nd }} \mathrm{T}$ and $62.3 \pm 16.5 \%$ during the $3^{\text {rd }} \mathrm{T}$. The figures for normal controls were $106 \pm 6.5 \%$. Functional PS was $43.8 \pm 13.3$ and $25.9 \pm 14.6 \%$ during the $2^{\text {nd }} \mathrm{T}$ and $3^{\text {rd }} \mathrm{T}$, respectively. The figures for normal controls were $97 \pm 24 \%$ ( $p<0.001$ compared with pregnant women). Free PS antigen did not change from the $2^{\text {nd }}$ to the $3^{\text {rd }} \mathrm{T}(\mathrm{p}=\mathrm{NS})$, however functional PS fell significantly from the $2^{\text {nd }}$ to the $3^{\text {rd }} \mathrm{T}$ ( $\mathrm{p}$ $<0.001$ ) and was significantly lower than free PS antigen in both trimesters $(p<0.001)$. Conclusions: Pregnancy is associated to a decrease in PS. This abnormality is more pronounced for functional PS than free PS antigen and functional PS falls progressively during pregnancy. These assays should not be used to screen for PS deficiency during pregnancy because they could lead to a misdiagnosis (Rev Méd Chile 2005; 133: 633-8).

(Key Words: Pregnancy trimesters; Protein S; Protein S deficiency)

Recibido el 15 de enero, 2004. Aceptado en versión corregida el 12 de abril, 2005.

Trabajo presentado en meeting del ASH 2001 y publicado como abstract en Blood.

Sección Hematología y Unidad de Medicina Fetal del Departamento de Ginecología y Obstetricia, Hospital Clínico de la Universidad de Chile. Laboratorio de Hematología, Clínica Alemana. Santiago, Chile.

aTecnólogo Médico.

bEstudiante, Facultad de Medicina de la Universidad de Chile.

Correspondencia a: Dra. Patricia Fardella B. Hospital Clíni-

co Universidad de Chile. Santos Dumont 999, Santiago.

Fax: (5-62) 6788248. E-mail: pfardella123@msn.com 
$\mathrm{E}^{1}$ embarazo normal es considerado un estado de hipercoagulabilidad durante el cual se produce un aumento de la incidencia de fenómenos tromboembólicos; estos eventos constituyen una de las principales causas de muertes maternas en países desarrollados y, de ellos, el tromboembolismo pulmonar sería la segunda causa $^{1,2}$.

Los mecanismos fisiopatológicos involucrados en la hipercoagulabilidad son la estasia de las venas pélvicas y de extremidades inferiores por compresión uterina, cambios en los mecanismos hemostáticos como el aumento de los niveles de factores de coagulación, aumento de la generación de trombina, inhibición de la fibrinólisis y disminución de las proteínas anticoagulantes naturales $^{3-5}$.

Las proteínas C y S (PC y PS) son anticoagulantes naturales que inactivan al factor Va y factor VIIIa e inhiben la acción del inhibidor del activador del plasminógeno (PAI). La PS que actúa como cofactor de la PC, se encuentra en el plasma en dos formas, una libre que es la forma funcional y otra unida a una proteína conocida como $\mathrm{C} 4 \mathrm{~b}$ binding protein (C4BP) que es inactiva ${ }^{6}$. Comunicaciones recientes señalan que la PS juega un rol favorecedor de la fibrinólisis, por una menor activación de TAFI (inhibidor de la fibrinólisis activado por trombina) a través de mecanismos dependientes e independientes de PC activada ${ }^{7}$.

La deficiencia de estas proteínas puede ser adquirida o heredada en forma autosómica dominante $^{8}$. La frecuencia de trombosis durante la gestación en pacientes portadores de deficiencia de estas proteínas aumenta y se estima entre 7 y $17 \%{ }^{9}$, sin embargo, el diagnóstico durante el embarazo es difícil, por los cambios fisiológicos antes descritos y porque no están definidos los valores normales en esta condición, presentando la PS funcional las mayores variaciones ${ }^{10-13}$. El objetivo de este estudio fue comparar los valores de PS libre determinada por dos métodos diferentes durante el $2^{\circ}$ y $3^{\text {er }}$ trimestre (T) del embarazo normal.

\section{MATERIAL Y MÉTODOS}

Estudiamos, en forma prospectiva, 44 embarazadas normales, cuyos controles de embarazo se realizaron en la Unidad de Medicina Fetal del Departamento de Obstetricia y Ginecología del Hospital Clínico de la Universidad de Chile. Se excluyeron del análisis de resultados 3 pacientes, por presentar trastornos de coagulación y anemia.

Se incluyeron pacientes sanas, con un embarazo único, entre 16 y 39 años de edad, sin historia personal ni familiar de trombosis, sin hipertensión arterial, no fumadoras, sin patologías médicas coexistentes, sin ingestión de fármacos a excepción de vitaminas y que cursaron un embarazo con un feto estructuralmente normal.

Se excluyeron pacientes que desarrollaran alguna patología durante el embarazo, aparición de trombosis, interrupción o término del embarazo antes de las 37 semanas y presencia de trastornos de coagulación. Todas las pacientes firmaron un consentimiento informado, aprobado por el comité de ética del Hospital.

Las determinaciones se realizaron durante el $2^{\circ} \mathrm{T}$ (22-24 semanas) y en el $3^{\text {er }} \mathrm{T}$ (29-38 semanas). Se extrajo sangre de una vena del antebrazo, utilizando tubos con citrato de sodio al 3,8\% con una relación 9:1. Las muestras para determinación de PS fueron puestas en hielo y centrifugadas, posteriormente se congelaron a $-20^{\circ} \mathrm{C}$ hasta ser procesadas. Se realizó además, hematocrito, hemoglobina, recuento de leucocitos, recuento de plaquetas, tiempo de tromboplastina parcial activado (TTPA), tiempo de protrombina (TP) y niveles de fibrinógeno. La PS libre se determinó por método de coagulación utilizando reactivos Dade Berhing, en un equipo semiautomático Berhing Fibrintimer y por técnica de ELISA con un reactivo Corgenix, que utiliza un anticuerpo monoclonal específico para proteína S libre (Free Protein S Antigen, Grifols) en un equipo Triturus.

Los valores normales se obtuvieron de los controles normales utilizados por el laboratorio, de sexo femenino y rango de edad semejante al grupo de estudio.

Estadística. Se analizaron ambos grupos realizando promedios, desviación estándar del promedio (DE) y test de t de student no pareados para comparar ambos trimestres del embarazo y los normales. 


\section{RESULTADOs}

Se excluyeron del estudio 3 embarazadas, 2 por presentar trombocitopenia (114 y 107 x 109/L) y una por anemia (hemoglobina $<8 \mathrm{~g} / \mathrm{dL}$ ).

No se observaron diferencias significativas entre las embarazadas de $2^{\circ}$ y $3^{\text {er }} \mathrm{T}$ en cuanto a edad, paridad, número de abortos espontáneos previos, hematocrito, hemoglobina, recuento de plaquetas y leucocitos, TTPA y TP. Los niveles de fibrinógeno durante el $2^{\circ} \mathrm{T}$ fueron significativamente más bajos que en los controles normales, con un $\mathrm{p}<0,02(2,33 \pm 0,32 \mathrm{~g} / \mathrm{L}$ versus $2,67 \pm 0,64$ $\mathrm{g} / \mathrm{L})$. Los niveles de fibrinógeno entre normales y embarazadas de $3^{\text {er }} \mathrm{T}$ no muestran diferencias significativas $(2,67 \pm 0,64 \mathrm{~g} / \mathrm{L}$ versus $2,65 \pm 0,66 \mathrm{~g} /$ L). Los parámetros clínicos y de laboratorio en embarazadas de $2^{\circ}$ y $3^{\text {er }} \mathrm{T}$ se muestran en la Tabla 1.

La PS libre realizada por ambos métodos, tanto en el $2^{\circ}$ como $3^{\text {er }} \mathrm{T}$ fue significativamente menor que en normales $(p<0,001)$. La PS por coagulación bajó en forma significativa desde el $2^{\circ} \mathrm{T}$ $(43,8 \pm 13,3 \%)$ al $3^{\text {er }} \mathrm{T}(25,9 \pm 14,6 \%)$ con un $p$ $<0,001$, sin embargo esto no se observa con la PS por ELISA $\left(2^{\circ} \mathrm{T} \quad 65,8 \pm 18,3 \%\right.$ versus $3^{\text {er }} \mathrm{T}$ $62,3 \pm 16,5 \%$ ). La PS por coagulación fue significativamente más baja que la PS por ELISA en ambos trimestres del embarazo ( $p<0,001)$. Los resultados se muestran en la Tabla 2.

Tabla 1. Parámetros clínicos y de laboratorio en embarazadas de $2^{\circ}$ y $3^{\text {er }}$ trimestre

\begin{tabular}{|lcc|}
\hline & $\begin{array}{c}2^{\circ} \mathrm{T}(\mathrm{n}=20) \\
(\text { promedio } \pm \mathrm{DS})\end{array}$ & $\begin{array}{c}3^{\text {er }} \mathrm{T}(\mathrm{n}=21) \\
\text { (promedio } \pm \mathrm{DS})\end{array}$ \\
\hline Edad & $29,6 \pm 4,4$ & $26,3 \pm 5,7$ \\
Paridad & 1,55 & 0,9 \\
Abortos espontáneos & 0,4 & 0,3 \\
Hematocrito (\%) & $33,5 \pm 2,3$ & $35 \pm 2,7$ \\
Hemoglobina (g/dL) & $11,1 \pm 1,0$ & $11,5 \pm 0,8$ \\
Recuento leucocitos (x 109/L) & $10,07 \pm 1,90$ & $9,98 \pm 2,1$ \\
Recuento de plaquetas (x 109/L) & $233 \pm 46$ & $220 \pm 48$ \\
Fibrinógeno (g/L) & $2,33 \pm 0,32^{*}$ & $2,65 \pm 0,66^{*}$ \\
TTPA (seg) & $27,3 \pm 2,6$ & $28,6 \pm 2,7$ \\
TP (seg) & $12,4 \pm 0,8$ & $12,2 \pm 0,9$ \\
\hline
\end{tabular}

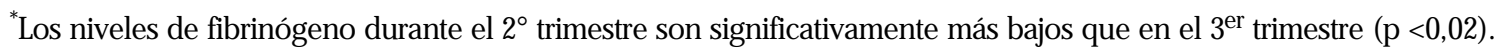

Tabla 2. Niveles de PS libre por coagulación y por ELISA

\begin{tabular}{|lccc|}
\hline & $\begin{array}{c}2^{\circ} \mathrm{T}(\mathrm{n}=20) \\
(\text { promedio } \pm \mathrm{DE})\end{array}$ & $\begin{array}{c}3^{\text {er }} \mathrm{T}(\mathrm{n}=21) \\
(\text { promedio } \pm \mathrm{DE})\end{array}$ & $\begin{array}{c}\text { Controles }(\mathrm{n}=19) \\
\text { (promedio } \pm \mathrm{DE})\end{array}$ \\
\hline PS por coagulación (\%) & $43,8 \pm 13,3$ & $25,9 \pm 14,6$ & $97 \pm 24$ \\
& $(15-65)$ & $(1-56)$ & $(70-150)$ \\
PS por ELISA (\%) & $65,8 \pm 18,3$ & $62,3 \pm 16,5$ & $106 \pm 6,5$ \\
& $(39-109)$ & $(39-109)$ & $(89-123)$ \\
\hline
\end{tabular}

Los niveles de PS libre por coagulación y por ELISA son significativamente más bajos que en controles durante el $2^{\circ}$ y $3^{\text {er }} \mathrm{T}$ del embarazo $(\mathrm{p}<0,001)$. Los niveles de PS libre por coagulación descienden en forma significativa desde el $2^{\circ}$ al $3^{\text {er }}$ trimestre del embarazo $(p<0,001)$. Los niveles de PS libre por coagulación son significativamente más bajos que los de PS por ELISA en el $2^{\circ}$ y $3^{\text {er }}$ trimestre del embarazo $(p<0,001)$. 


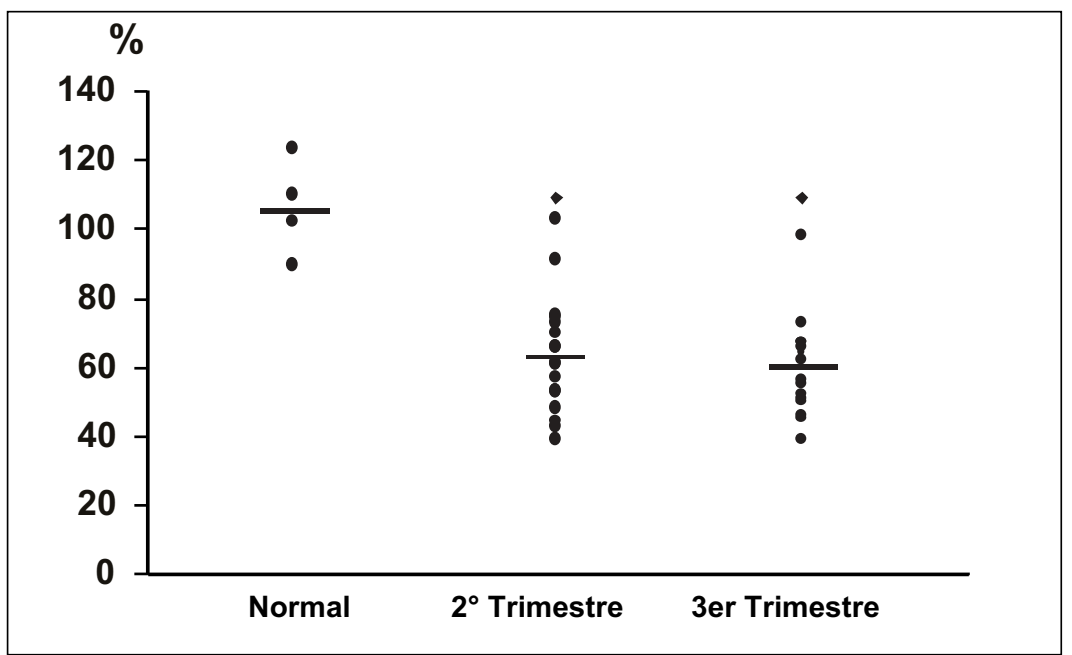

FIgURA 1. La PS libre por ELISA $2^{\circ}$ y $3^{\text {er }} \mathrm{T}$ es más baja que en normales $(p<0,001)$. No hay diferencias entre $2^{\circ} \mathrm{y}$ $3^{\mathrm{er}} \mathrm{T}$.
FiguRA 2. La PS libre por coagulación en $2^{\circ}$ y $3^{\text {er }} \mathrm{T}$ es significativamente más baja que en normales $(p<0,001)$ y desciende en forma significativa del $2^{\circ} \mathrm{T}$ al $3^{\mathrm{er}} \mathrm{T}(\mathrm{p}<0,001)$.

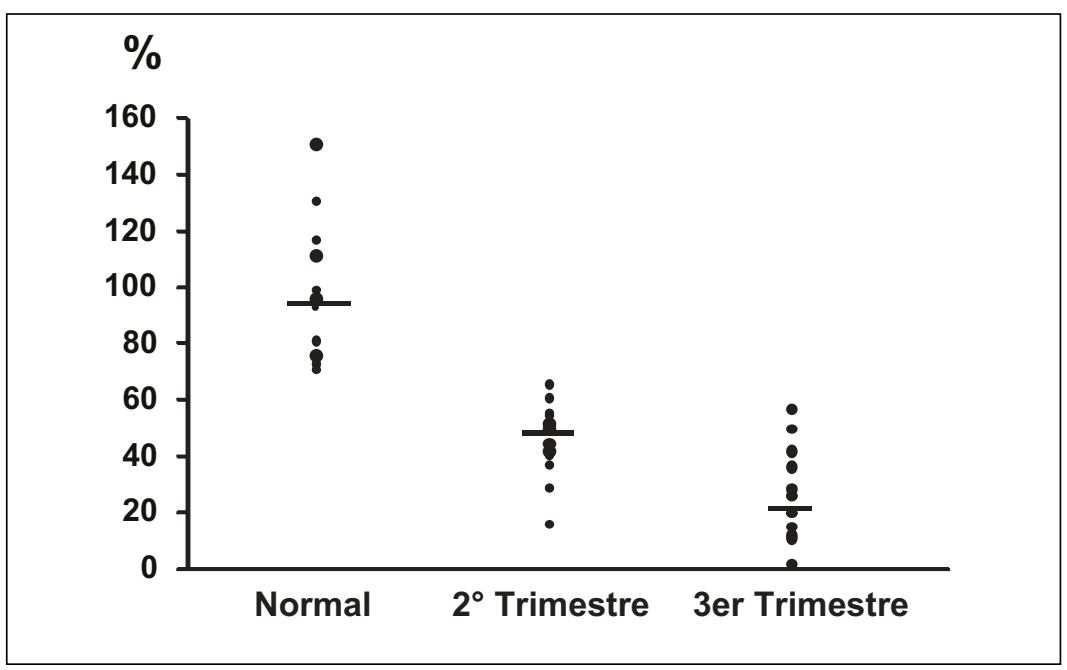

La Figura 1 muestra los niveles de PS por ELISA en $2^{\circ}$ y $3^{\text {er }} \mathrm{T}$ en relación a los controles normales, observándose que es significativamente más baja, sin embargo no se observa descenso entre un trimestre y otro.

La Figura 2 muestra los niveles de PS por método de coagulación, observándose valores significativamente más bajos que los normales en ambos trimestres y un descenso significativo entre el $2^{\circ}$ y $3^{\text {er }} \mathrm{T}$.

No observamos ningún episodio de trombosis clínica en estas pacientes durante el embarazo ni el puerperio.

\section{DisCUSIÓN}

Durante el embarazo normal se produce un balance hemostático en sentido de la hipercoagulabilidad, con el objeto de disminuir las complicaciones hemorrágicas durante el parto. Se puede observar un aumento de la generación endógena de trombina, resistencia a la proteína $\mathrm{C}$ adquirida, aumentos progresivos de los niveles de muchos factores de coagulación entre ellos el fibrinógeno, lo que conduce a un discreto acortamiento del TTPA y del tiempo de protrombina. Se observa además aumento de dímero-D y del fragmento 
$1+2$ de la protrombina que indican la presencia de un estado de hipercoagulabilidad ${ }^{14-16}$.

La actividad fibrinolítica está disminuida durante el embarazo, debido a un aumento del inhibidor del activador del plasminógeno 1 (PAI1) producido por la célula endotelial y del PAI-2 producido por la placenta ${ }^{14}$. La actividad anticoagulante natural también se encuentra alterada y está bien establecida una disminución de los niveles de PS libre o funcional. En relación a la proteína $C$ las variaciones durante el embarazo no están definidas, ya que existen reportes donde sus niveles son normales y otros que señalan que disminuye durante la gestación ${ }^{10,13,17}$. En cuanto a la antitrombina III no se observan variaciones en sus niveles durante el embarazo ${ }^{18}$.

Estas alteraciones de la hemostasia son progresivas en el transcurso del embarazo y continúan hasta el puerperio, aumentando el riesgo de fenómenos trombóticos en mujeres sanas. En mujeres portadoras de deficiencias de PS, PC y antitrombina III la incidencia de trombosis es 8 veces mayor durante el embarazo ${ }^{19}$. Por estas razones, en pacientes sin antecedentes de trombofilia que presentan trombosis durante el embarazo, es muy importante saber si las pruebas realizadas en ese momento tienen validez para definir un diagnóstico o sólo son fenómenos ocasionados por una condición fisiológica.

Es conocido que los niveles de PS libre disminuyen durante el embarazo, pero la magnitud de estos cambios varía según el método que se utilice para determinarla ${ }^{8,10,20}$. Como se señaló anteriormente, en el plasma existe un equilibrio entre la PS libre o funcionalmente activa y la PS unida a una proteína plasmática que une a la fracción del complemento $\mathrm{C} 4 \mathrm{~b}$ (C4b Binding Protein); en estas condiciones la PS es inactiva. La C4BP es una proteína de alto peso molecular que une a la fracción C4b del complemento y a la PS, en forma no competitiva. La C4BP tiene 6 a 8 unidades idénticas que unen $\mathrm{C} 4 \mathrm{~b}$ y una cadena beta única que es el sitio de unión de la PS. En el plasma se han descrito más de una forma de C4BP y es así como una forma de peso molecular más alto, que constituye alrededor de $80 \%$ de la proteína, sería la que puede unir a la PS en un sitio de unión diferente al de $C 4 b^{11}$.

Como una forma de explicar la disminución de la PS libre durante el embarazo se han realizado determinaciones de C4BP en embarazadas, observándose en algunos trabajos que no hay diferencias con respecto a las mujeres normales, postulando que el descenso de PS libre sería real ${ }^{11}$; este hecho se ve apoyado por algunos reportes que indican que el uso de anticonceptivos orales producen una disminución de PS libre, sin observar una modificación en los niveles de C4BP, atribuyendo los cambios en la PS al cambio en los niveles hormonales ${ }^{21}$; sin embargo hay otros que indican que los niveles de C4BP se van elevando en el transcurso del embarazo, lo que podría explicar la disminución de los niveles por un aumento de la unión a proteína ${ }^{22}$.

En nuestro estudio observamos que la PS libre es significativamente más baja que en los normales, al realizarla con ambos métodos; no realizamos determinaciones de C4BP, por lo que no sabemos el comportamiento y por tanto la influencia de esta proteína en los niveles de PS libre en nuestras pacientes. Observamos además que los niveles de PS fueron significativamente más bajos cuando se realizaron por método de coagulación que con el método de ELSA en ambos trimestres, indicando que en el primero estarían interfiriendo otros elementos de la coagulación. Además con el método de coagulación observamos un descenso significativo de los niveles de PS libre entre el $2^{\circ} \mathrm{T}$ y $3^{\text {er }} \mathrm{T}$. Estos resultados son corroborados por el estudio de Lefkowitz et $\mathrm{al}^{20}$ y Fernández et $\mathrm{al}^{23}$ los cuales lo explican por el aumento de factores de coagulación, entre ellos factor VIII, factor $\mathrm{V}$ y fibrinógeno, que ocurren durante el embarazo y que interferináan con los exámenes de coagulación pero no con las determinaciones antigénicas que utiliza el método de ELSA ${ }^{24,25}$. En este grupo no se realizó niveles de factor $V$ ni factor VIII, por lo que, a pesar de que los resultados son similares no podemos asumir que ésta sea la explicación en nuestro grupo. Las determinaciones de fibrinógeno en nuestras pacientes no concuerdan con esta explicación, ya que observamos que en el $2^{\circ} \mathrm{T}$ presentan niveles de fibrinógeno significativamente más bajos que los normales y en el $3^{\text {er }} \mathrm{T}$ tienen niveles similares a los controles, por lo que esta explicación no sería válida para nuestro grupo.

A pesar de no poder dar una explicación fisiopatológica clara para nuestros hallazgos, los resultados de ambos métodos son similares a los comunicados en la literatura. 
El diagnóstico de déficit de PS no debería realizarse durante el $2^{\circ}$ y $3^{\text {er }}$ trimestre del embarazo ya que puede llevar a un diagnóstico erróneo, ya que los niveles de PS libre en esta población de embarazadas normales se encuentra en rangos

\section{REFERENCIAS}

1. JoIst JH. Hypercoagulability: introduction and perspective. Semin Thromb Hemost 1990; 16: 151-7.

2. De SwerT M. Thromboembolism. Clin Lab Haematol 1985; 14: 643-60.

3. Wiman B, Csemiczky G, Marsk L, Robbe H. The fast inhibitor of tissue type plasminogen activator in plasma during pregnancy. Thromb Haemost 1984; 52: 124-6.

4. Pinto S, Abbate R, Rostagno C, Brun V, Rosati D, Neri SERNERI GG. Increased thrombin generation in normal pregnancy. Acta Europea Fertilitatis 1988; 19: 263-7.

5. Bremme K, Ostlund E, Almovist I, Heinonen K, Bцомваск M. Enhanced thrombin generation and fibrinolytic activity in normal pregnancy and puerperium. Obstet Gynecol 1992; 80: 123-37.

6. Dahıback B. Protein S and C4b-Binding protein: Components involved in the regulation of the protein $\mathrm{C}$ anticoagulant system. Throm Haemost 1991; 66: 49-61.

7. Mosnier LO, Mejers JMC, Bouma BN. The role of protein $\mathrm{S}$ in the activation of thrombin activable fibrinolysis inhibitor (TAFI) and regulation of fibrinolysis. Thromb Haemost 2001; 86: 1040-6.

8. BICK RL Prothrombin G20210A mutation, antithrombin, heparin cofactor II, protein $C$ and protein S defects. Hematol Oncol Clin NAm 2003; 17: 9-36.

9. Morrison AE, WaLKer ID, BLACK WP. Protein C deficiency presenting as deep vein thrombosis in pregnancy. Br J Obstet Gynaecol 1988; 95: 1007-10.

10. Faught W, Garner P, Jones G, Ivey B. Changes in protein $\mathrm{C}$ and protein $\mathrm{S}$ levels in normal pregnancy. Am J Obstet Gynecol 1995; 172: 147-50.

11. Comp PC, Thurnau GR, Welsh J, Esmon C. Functional and inmunologic protein $\mathrm{S}$ are decreased during pregnancy. Blood 1986; 68: 881-5.

12. WARwick $R$. Changes in protein $C$ and free protein $\mathrm{S}$ during pregnancy and following hysterectomy. Royal Soc Med 1989; 82: 671-5.

13. Lao TT, Yuen PMP, Yin JA. Protein $S$ and protein $C$ levels in Chinese women during pregnancy, delivery and the puerperium. Br J Obstet Gynaecol 1989; 96: 167-70. establecidos como patológicos para la población general. No tenemos certeza si este fenómeno favorece la hipercoagulabilidad en el embarazo o es sólo el resultado de otras alteraciones de la coagulación propias de esa condición.

14. HELLGREN M. Hemostasis during nomal pregnancy and puerperium. Semin Thromb Hemost 2003; 29: 125-30.

15. Francalanci I, Comeglo P, Alessandrello L, Cellai AP, Fedi S, ParReti E ET al. D-dimer concentrations during normal pregnancy, as measured by ELSA. Thromb Res 1995; 78: 399-405.

16. Comeglo P, Fedi S, Alessandrelo L, Cemai AP, Chiarantini E, Prisco D et al. Blood Clotting Activation during normal pregnancy. Thromb Res 1996; 84: 199-202.

17. Oruc S, Saruc M, Koyuncu FM, Ozdemir E. Changes in the plasma activities of protein $C$ and protein $S$ during pregnancy. Aust NZJ Obstet and Gynaecol 2000; 40: 448-50.

18. Jensen R, FRitsma GA. Thrombosis risk testing. Clinical Hemostasis Review 1998; 12: 1-17.

19. Friederich PW, Simioni P, Zanardi SS, Huisman MV, Kirdt I, PRANDoni P et AL. Frequency of pregnancyrelated venous thromboembolism in anticoagulant factor-deficient women: implications for prophylaxis. Ann Intern Med 1996; 125: 955-60.

20. Lefrowitz JB, Clarke SH, Barbour LA. Comparison of protein $\mathrm{S}$ functional and antigenic assays in normal pregnancy. Am J Obstet Gynecol 1996; 175: 657-60.

21. Boerger LM, Morris PC, Thurnan GR, Esmon CT, Comp PC. Oral contraceptives and gender affect protein S status. Blood 1987; 69: 692-4.

22. Malm J, Laurell M, Dahlback B. Changes in the plasma level of Vitamin K-dependent proteins C and $\mathrm{S}$ and of $\mathrm{C} 4 \mathrm{~b}$ - binding protein during pregnancy and oral contraceptives. Br J Haematol 1988; 68: 437-43.

23. Fernández JA, Estelís A, Gilabert J, España F, Aznar $J$. Functional and immunogenic protein $S$ in normal pregnant women and full term newborns. Thromb Haemost 1989; 61: 474-8.

24. Stirling Y, Woolf L, North WRS, Seghatchian MJ, MEAD TW. Hemostasis in normal pregnancy. Throm Haemost 1984; 52: 176-82.

25. Deitacher SR, Volner JS, Kottke-Marchant K. Activated protein $\mathrm{C}$ resistance (APC-R) and elevated factor VIII (FVIII) activity associated interference in functional protein S assays. Blood 1998; 92: 131b. 